\title{
The Application of Reactive Polydopamine Thin Films for the Functionalization of Tissue Culture Plastic with Alkaline Phosphatase
}

\author{
Jason P Mansell*, Kamal Dahab and Thomas Cyprien \\ Department of Applied Sciences, University of the West of England, UK \\ *Corresponding author: Jason P Mansell, Department of Applied Sciences, University of the West of England, Bristol, \\ BS16 1QY, UK
}

ARTICLE INFO

Received: 幽 April 29, 2020

Published: May 07, 2020

Citation: Jason P Mansell, Kamal Dahab, Thomas Cyprien. The Application of Reactive Polydopamine Thin Films for the Functionalization of Tissue Culture Plastic with Alkaline Phosphatase. Biomed J Sci \& Tech Res 27(3)-2020. BJSTR. MS.ID.004511.

Keywords: Alkaline Phosphatase; Polydopamine; Functionalization; Biomaterials

\author{
ABSTRACT
}

Improving the biological performance of implantable bone biomaterials is predicted to enhance their longevity. Given that aseptic loosening of total joint replacements is the largest cause of failure it is pertinent to explore ways in which surfaces could be functionalised with suitable bioactive agents to bolster early osseointegration and union with host bone tissue. Before moving to more costly implantable materials, such as titanium, it is pragmatic to identify inexpensive materials to help establish proof-ofconcept. We therefore chose tissue culture plastic and functionalised this material with alkaline phosphatase (ALP) given its essential role in bone collagen mineralisation. To ensure a reliable, robust attachment we used polydopamine (PDA) as the capturing platform. Herein we provide evidence for the facile fabrication of an ALP-functionalised material that will help inform future studies towards the development of superior implantable technologies.

\section{Short Communication}

Aseptic loosening continues to be the major cause of total joint replacement (TJR) failure [1,2]. In England and Wales alone approximately $7-8 \%$ of all TJR's will fail within the lifetime of the patient [3]. This represents a significant burden to our healthcare providers. Enhancing the longevity of TJR's is clearly warranted to minimise the extent of aseptic failure. One way in which this could be realised is to coat the material surface with bioactive agents that are key to the process of bone matrix synthesis and mineralisation. If a mechanically robust tissue could be secured early at the bone-implant interface, then there is greater potential that the overall process of osseointegration would be bolstered and with-it improvements in prosthesis survivorship.

An essential component of bone collagen calcification is alkaline phosphatase (ALP) which serves to ensure a source of inorganic phosphate from pyrophosphate. The significance of ALP in overall bone health is supported by the clinical correlate of hypophosphatasia wherein missense mutations in the ALP gene result in a skeletal phenotype resembling rickets and osteomalacia
[4]. Since ALP is inextricably linked to bone matrix calcification, tethering ALP to bone biomaterials may be one way towards improving TJR longevity. To appeal to the implant manufacturers the route to ALP immobilisation should be facile and amenable to large scale production. To this end we have turned our attention to polydopamine (PDA) thin films for the capture of ALP. PDA thin films represent highly versatile reactive platforms for the facile fabrication of novel (bio)materials [5-7]. Indeed, PDA coatings are currently regarded as "start-of-the-art" functional supports with wide ranging applications [8]. Simply steeping materials in mildly alkaline solutions of dopamine hydrochloride (DHC) results in the spontaneous polymerisation to PDA, a brown-black complex analogous to the formation of eumelanin [9]. The tenacious binding of PDA to multiple substrates lends itself for further modification by exploiting the plentiful amino, quinone and catechol moieties [5]. Schiff-base and Michael addition reactions, for example, afford peptide and protein conjugation of PDA coatings in generating a wide spectrum of functional surfaces.Using an inexpensive tissue culture plastic (TCP) model we present evidence for the rapid and 
facile immobilisation of ALP using PDA which will help inform further studies towards the fabrication of superior TJR technologies.

\section{Materials and Methods}

All reagents were from Sigma (Poole, Dorset, UK). Bovine intestinal ALP (Sigma cat. P0114) was diluted to $1 \mathrm{KU} / \mathrm{ml}$ using $50 \%$ glycerol in a neutral buffer comprising of $10 \mathrm{mM}$ HEPES $(\mathrm{pH}$ 7.4), $1 \mathrm{mM}$ magnesium chloride and $100 \mu \mathrm{M}$ zinc acetate. Stocks were kept refrigerated throughout the duration of the study. For the ALP assay a di-tris salt of para-nitrophenylphosphate (p-NPP, Sigma cat. N3254) was used.

Functionalisation of tissue culture plastic (TCP) - ALP was diluted to $2 \mathrm{U} / \mathrm{ml}$ in $20 \mathrm{mM}$ Trizma (pH 9.5). A pH of 9.5 was informed from pilot studies to minimise ALP adsorption to the test surface. Aliquots $(0.9 \mathrm{ml})$ of this working ALP solution were dispensed into the wells of 24-well plates and served as adsorption controls. Unless stated otherwise a portion of the same ALP solution was dispensed onto solid dopamine hydrochloride (DHC, Sigma cat. H8502) to give a final concentration of $2 \mathrm{mg} / \mathrm{ml}$. Within a minute of gentle mixing $0.9 \mathrm{ml}$ of the ALP-DHC solution was applied to each of the test wells. Unless stated otherwise solutions were left for 30 minutes at room temperature.

Alkaline phosphatase (ALP) assay - Once experiments had run their course reaction solutions were aspirated and the wells rinsed thrice with distilled water dispensed from a squeezy bottle. Excess water was removed from the plates with shaking. An ALP substrate buffer ( $\mathrm{pH}$ 10.3) was prepared by combining equimolar $(0.1 \mathrm{M})$ sodium carbonate and sodium bicarbonate as instructed [10]. Briefly $700 \mathrm{ml}$ of sodium carbonate was blended with $300 \mathrm{ml}$ of sodium bicarbonate to achieve the desired $\mathrm{pH}$. This buffer was supplemented with $\mathrm{MgCl} 2$ to a final concentration of $1 \mathrm{mM}$. Within minutes of intended use, para-nitrophenylphosphate (p-NPP) was dissolved to a final concentration of $5 \mathrm{mg} / \mathrm{ml}$ in the ALP buffer and $0.3 \mathrm{ml}$ dispensed into each of the wells. Plates were left to incubate for 30 minutes at room temperature. Sample aliquots $(0.1 \mathrm{ml})$ were then transferred to 96 -well plates and the absorbance at $405 \mathrm{~nm}$ taken using a multiwell plate reader (FLUOstar OPTIMA, BMG labtech) operated from a PC using MARS software. An ascending series of p-nitrophenol (p-NP, 0-500 $\mu$ M, Sigma cat. 1048) prepared in the ALP buffer enabled extrapolation of $\mathrm{p}$-NP concentration in the test samples.

Statistical analysis - Unless stated otherwise, results are expressed as the mean plus the standard deviation (SD). Data were subjected to either paired or unpaired t-tests (2-tailed) to compare means of functionalised and control surfaces. P-values of $<0.05$ were considered statistically significant. All experiments were repeated at least twice with a minimum of 4 technical repeats for each of the different groups.

\section{Results}

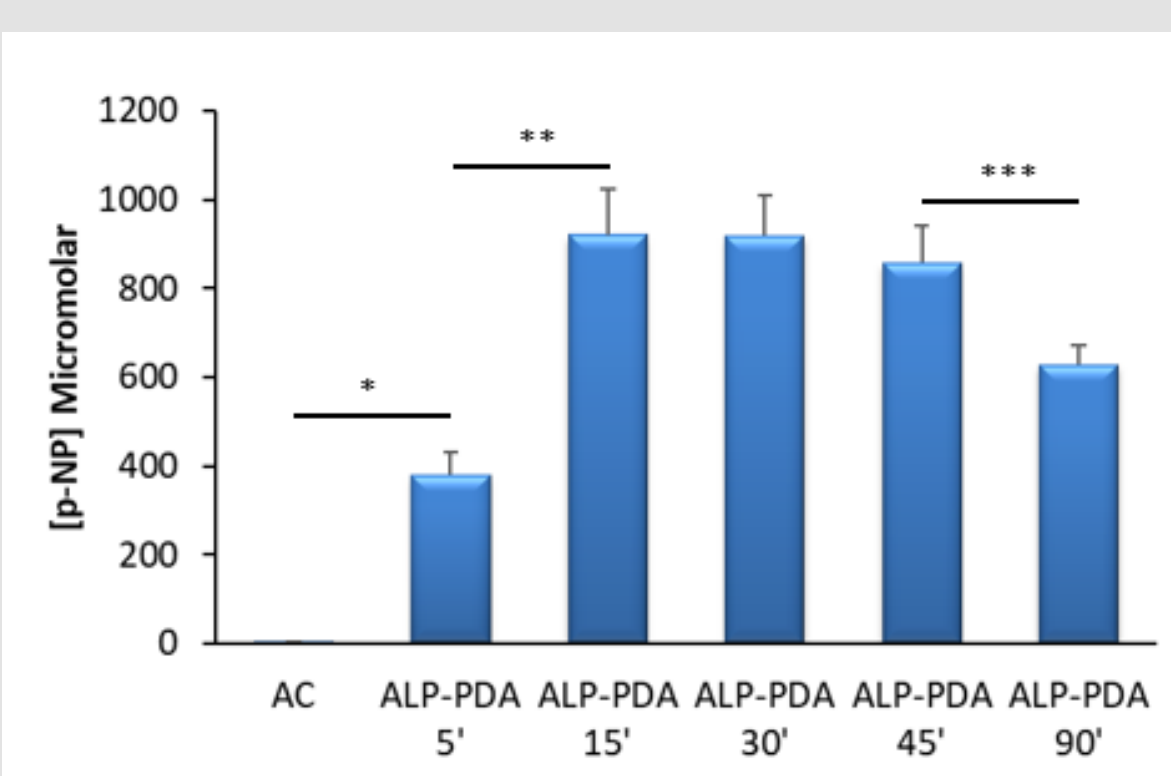

Figure 1: Successful immobilisation of alkaline phosphatase (ALP) using polydopamine (PDA) - Mulltiwell (24-well) tissue culture plastic (TCP) plates were either exposed to ALP alone (AC) or in combination with dopamine hydrochloride (2mg/ $\mathrm{ml}$ ) under alkaline conditions (20mM Tris, $\mathrm{pH} 9.5)$ at room temperature. Detection of ALP was via the quantification of p-nitrophenol ( $\mathrm{p}-\mathrm{NP}$ ) from p-nitrophenylphosphate. Capture of ALP at TCP by PDA was clearly evident from 5 minutes $\left({ }^{*} \mathrm{p}<0.001\right)$ compared to the adsorption control $(\mathrm{AC})$. The increase in ALP immobilisation continued over the next 10 minutes of TCP treatment $\left({ }^{* *} \mathrm{p}<0.001\right)$ and remained fairly constant for a further 30 minutes. However, extending the treatment period between 45 and 90 minutes led to a clear reduction in ALP activity (*** $p=0.006)$. For each bar the data are the mean values from 4 replicates plus the SD. 
ALP is readily immobilised using PDA thin films - We find that ALP could be deposited at TCP surfaces within a matter of minutes when the enzyme was combined with DHC in a one-pot formulation (Figure 1). The overall ALP activity for treated TCP surfaces was similar over a 45-minute exposure at room temperature after which it began to fall. Throughout the course of the experiments we saw little or no ALP activity when the enzyme was applied to TCP in the absence of DHC. These findings confirm that the enzyme was associated with the PDA matrix and not merely adsorbed to the TCP surface. As detailed by us previously [11], confirmation of
PDA deposition was via reaction of functionalised surfaces with bicinchoninic acid giving a violet chromogen (data not shown).

Altering the DHC concentration affects ALP capture at TCP Initially we prepared ALP in a $2 \mathrm{mg} / \mathrm{ml}$ solution of DHC, the most widely used concentration for surface functionalisations. Halving the DHC concentration appeared to improve TCP functionalisation with ALP but it did not reach statistical significance. Doubling the DHC concentration had a clear and significant impact upon ALP immobilisation leading to surfaces with less enzyme activity (Figure 2).

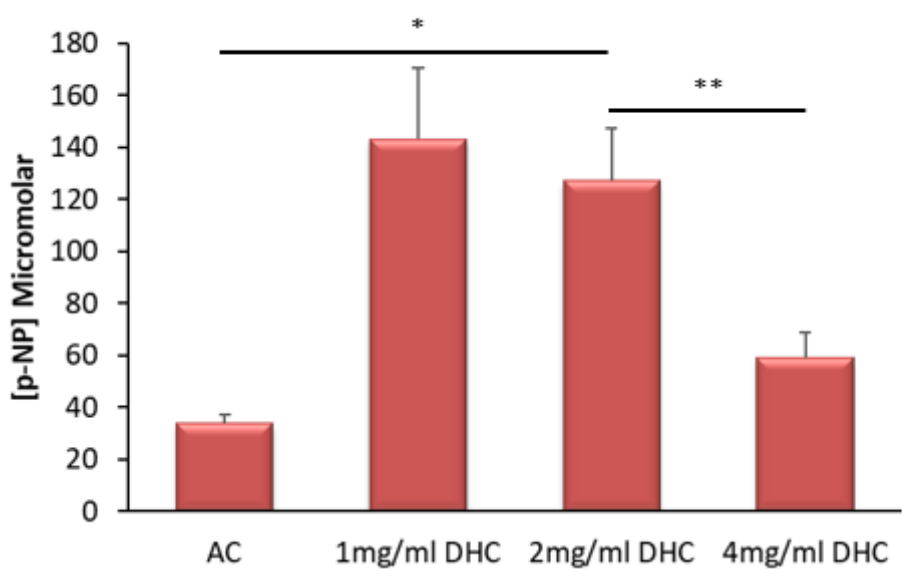

Figure 2: Dopamine hydrochloride (DHC) concentration affects alkaline phosphatase (ALP) immobilisation at tissue culture plastic (TCP) - An alkaline solution (20mM Tris, pH 9.5) of ALP was dispensed into the wells of 24-well TCP plates, either alone $(\mathrm{AC})$, or in the presence of different concentrations of DHC $(1-4 \mathrm{mg} / \mathrm{ml})$. Plates were left for 30 minutes at room temperature. Detection of ALP was via the quantification of p-nitrophenol (p-NP) from p-nitrophenylphosphate. As anticipated applying ALP to TCP using DHC at $2 \mathrm{mg} / \mathrm{ml}$ led to a reliable and significant $\left({ }^{*} \mathrm{p}<0.0001\right)$ immobilisation of ALP versus the adsorption control (AC). Whilst it seems that using DHC at $1 \mathrm{mg} / \mathrm{ml}$ might be better for ALP immobilisation compared to $2 \mathrm{mg} / \mathrm{ml}$ it did not reach statistical significance $(p=0.067)$. Increasing the DHC concentration to $4 \mathrm{mg} / \mathrm{ml}$ was associated with a significant $\left({ }^{* *} \mathrm{p}<0.0001\right)$ reduction in ALP immobilisation. Depicted are the pooled mean data from four independent experiments $(\mathrm{N}=16$ per bar) plus the SD.

ALP immobilisation by PDA results in a robust surface functionalisation - An important aspect of biomaterial modification is an assessment of coating stability. Given that TJR's are subjected to irrigation during implantation we thought it pertinent to determine if the ALP-PDA matrix could withstand washing and reuse. Herein we provide evidence that ALP-PDA could be washed with distilled water and the surfaces recused at least three times without any appreciable loss of enzyme activity (Figure 3).

ALP-PDA treated surfaces can be stored at $-20^{\circ} \mathrm{C}$ if immersed in an appropriate buffer - We next examined the stability of the ALP-PDA coating to 24 hours storage at $-20^{\circ} \mathrm{C}$. Early experiments indicated that storing functionalised surfaces "dry" was very detrimental with little or no enzyme activity remaining. However, if treated surfaces were immersed in either the enzyme diluent or substrate buffer there was a clear retention of enzyme activity (Figure 4).

\section{Discussion}

Enhancing the performance and longevity of TJR's continues to be a significant theme in contemporary biomaterials research. Coating devices with suitable biological agents may be one route to generating implantable technologies that are less likely to fail through loosening. To this end we chose ALP, an ectoenzyme essential for bone collagen calcification [4]. Whilst we have already shown that ALP can adsorb to titanium (Mansell et al. 2016), a widely used TJR material, a more secure attachment would be preferable to guarantee ALP persistence at the material surface. Recently Andrade and colleagues [12] grafted dimyristoylphosphatidic acid (DMPA) on to titanium using the Langmuir-Blodgett (LB) technique for the subsequent incorporation of ALP by exploiting the interaction of the ALP glycosylphosphatidylinositol hydrophobic anchor [13] with the adsorbed DMPA. Whilst the approach taken by Andrade et al. [12] delivered a surface finish capable of mineralisation it 
may not be the most pragmatic approach in future TJR design; the LB techniques is technically challenging and there is a reliance upon chloroform and methanol in DMPA grafting. It may be more appropriate to find simpler solutions so that they have greater appeal to implant manufacturers. We therefore chose PDA as the matrix to capture ALP.

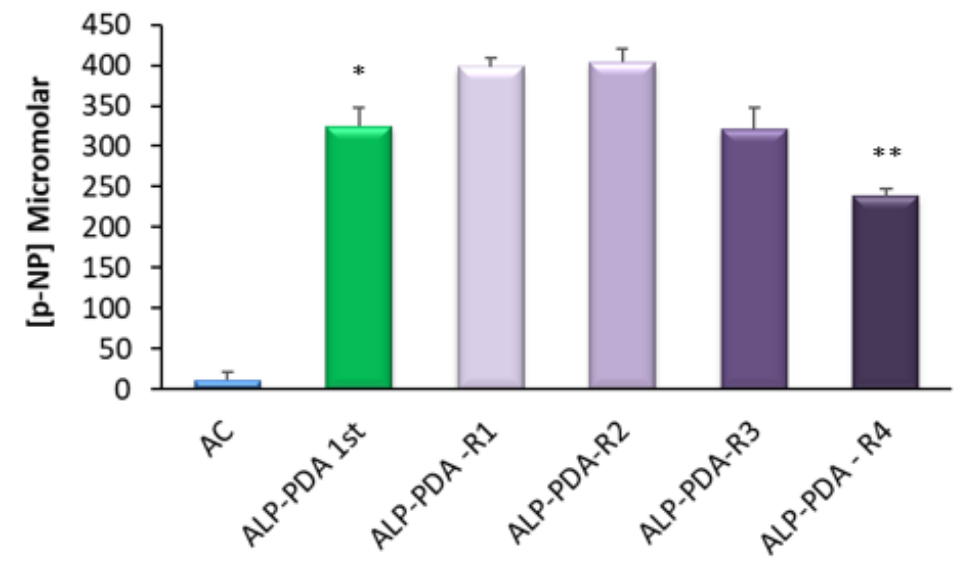

Figure 3: Evidence for the robust immobilisation of alkaline phosphatase (ALP) at tissue culture plastic (TCP) using polydopamine (PDA) - An alkaline solution (20mM Tris, pH 9.5) of ALP was dispensed into the wells of TCP 24-well plates, either alone (AC), or in combination with $2 \mathrm{mg} / \mathrm{ml}$ dopamine hydrochloride. Plates were left for 30 minutes at room temperature after which wells were rinsed thrice with distilled water. Detection of immobilised ALP was via the quantification of p-nitrophenol (p-NP) from p-nitrophenylphosphate. As anticipated the initial treatment of TCP with immobilised ALP (ALP-PDA 1st) resulted in an active surface finish $\left({ }^{*} \mathrm{p}<0.0001\right)$ compared to the adsorption control (AC). On completion of the ALP assay the wells were left for an hour, aspirated, rinsed and fresh ALP substrate applied. These reused wells (ALP-PDA-R1) show clear evidence for ALP persistence. When the process was repeated a second time (ALP-PDA-R2) the overall activity remained unchanged. The same wells were reused a third and fourth time, not an hour apart on these occasions but a day apart. Compared to the initial use there was a clear reduction in ALP activity with the fourth reuse $\left({ }^{* *} p=0.002\right)$. For each bar the data are the mean values from 4 replicates plus the SD.

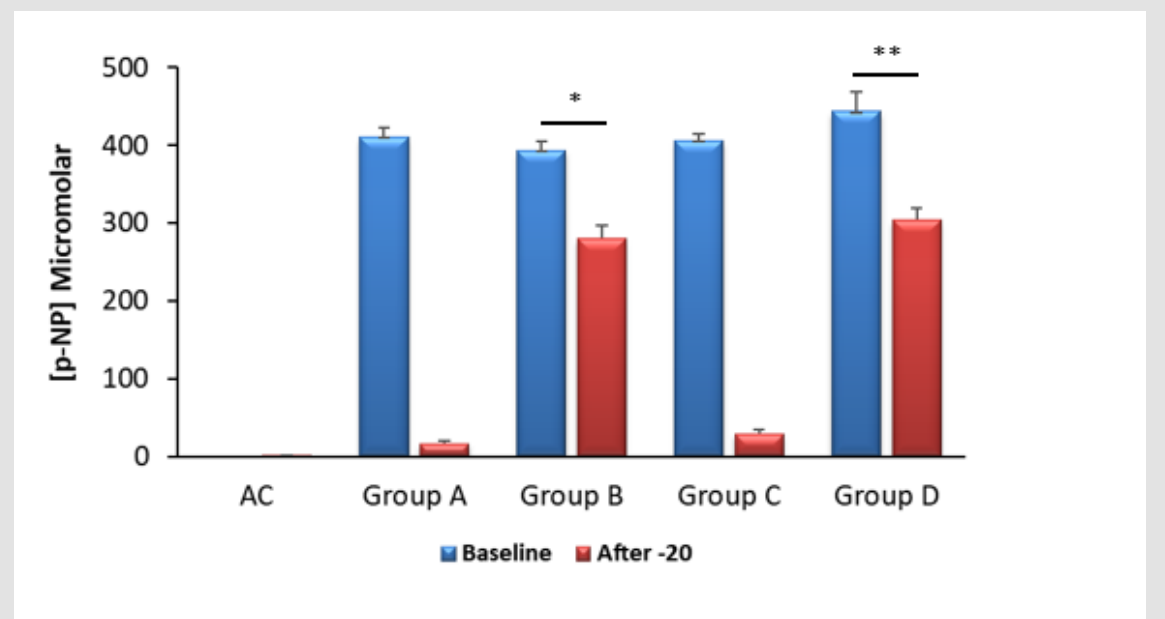

Figure 4: The stability of polydopamine (PDA) - immobilised alkaline phosphatase (ALP) to storage at $-20^{\circ} \mathrm{C}-\mathrm{An}$ alkaline solution (20mM Tris, pH 9.5) of ALP was applied to wells of 24-well tissue culture plates either alone (AC) or in combination with $2 \mathrm{mg} / \mathrm{ml}$ dopamine hydrochloride for 30 minutes at room temperature. Detection of ALP was via the quantification of p-nitrophenol (p-NP) from p-nitrophenylphosphate. After the baseline ALP assays had been completed the plates were split into four groups; for group A the wells were rinsed and emptied. The remaining substrate and products were left in situ for group B. For group C wells were rinsed and treated with $0.2 \mathrm{ml}$ of water whereas those in group D wells were rinsed and exposed to $0.2 \mathrm{ml}$ of the ALP diluent. All plates were then left at $-20^{\circ} \mathrm{C}$ for 24 hours after which a second ALP assay was performed to assess enzyme survivorship. It is clear that storing PDA-ALP "dry" (Group A) or in water (Group C) has a negative impact upon ALP activity. Conversely if PDA-ALP is stored frozen in the presence of p-NP and residual substrate (Group B) or a neutral ALP diluent (Group D) there is clear preservation of enzyme activity. However, there are modest, yet statistically significant reductions in ALP activity for groups $B\left({ }^{*} p<0.0001\right)$ and $D\left({ }^{* *} p<0.0005\right)$ after freezing. 
The formation of PDA thin films is substrate independent and forms rapidly by simply exposing the selected (bio)material for functionalisation in dilute, alkaline solutions of DHC [6]. Peptides and proteins react well with PDA forming covalent linkages between primary amino groups and oxidised catechol moieties of PDA via Michael addition and Schiff-base reactions [14-16]. We adopted a "one-pot" approach to surface functionalisation using inexpensive TCP to establish proof-of-concept before moving towards expensive implantable materials such as titanium. The use of PDA to tether ALP is not new; Nijhuis and co-workers (2013) used bovine intestinal ALP to functionalise titanium. They exposed samples to $81 \mathrm{U}$ of ALP ( $\sim 1 \mathrm{mg} / \mathrm{ml}$ of protein) for 16 hours. In our study we used $2 \mathrm{U}$ of bovine intestinal ALP and found that TCP could be functionalised within 45 minutes after which the surfaces were associated with far less enzyme activity. It is possible that the approach taken by Nijhuis et al. [17] may have compromised the final surface activity. Longer exposure times produce PDA films that are thicker and rougher [18] and may be prone to losses through even gentle material handling. Credence is given to this possibility considering our findings when using $4 \mathrm{mg} / \mathrm{ml}$ DHC; in comparison to using 1 or $2 \mathrm{mg} / \mathrm{ml}$ DHC the final surface finish had significantly less ALP activity associated with it. We have previously reported that raising the DHC concentration will increase the amount of PDA deposition [11]. It is unclear at present as to why higher DHC concentrations and/or longer steeping times compromise the ALP activity. It might be that the enzyme is adulterated during the polymerisation process. Collectively it may be wise to have much shorter incubation times to ensure a greater level of ALP activity at the material surface.

We next explored the stability of the PDA-ALP to irrigation and reuse. We chose this approach because medical implants are frequently irrigated during surgery. Herein we report that functionalised TCP could be washed and reused at least three times suggesting that ALP attachment by PDA was robust. We also considered storage of the functionalised surfaces to $-20^{\circ} \mathrm{C}$. It soon became apparent that leaving the samples "dry" in the freezer resulted in the loss of nearly all the enzyme activity. However, if the modified surfaces were treated with the ALP diluent and then frozen the resultant samples retained appreciable activity, approximately $70 \%$ of the baseline value. The same was true for samples frozen in ALP substrate buffer with p-NP and residual p-NPP present. Functionalised samples stored this way exhibited comparable enzyme activity for at least six months (data not shown).

\section{Conclusion}

Herein we provide evidence for the facile functionalisation of TCP with ALP using reactive PDA as the capturing matrix. Our findings have established proof-of-concept before moving towards the modification of titanium, a widely used bone implant material. We advise short surface exposure times ( $\sim 30$ minutes) to achieve maximal ALP activity. Likewise, we recommend that functionalised surfaces be stored in the presence of an ALP-compatible buffer to preserve enzyme activity.

\section{Acknowledgement}

The authors would like to thank the technical team at the Centre for Research in Biosciences, University of the West of England, for their help during this investigation.

\section{Conflict of Interest}

There are no conflicts to declare.

\section{References}

1. Sadoghi P, Liebensteiner M, Agreiter M, Leithner A, Bohler N, et al. (2013) Revision surgery after total joint arthroplasty: A complication-based analysis using worldwide arthroplasty registers. J Arthroplast. 28: 1329-1332.

2. Schroer WC, Berend KR, Lombardi AV, Barnes CL, Bolognesi MP, et al. (2013) Why are total knees failing today? Etiology of total knee revision in 2010 and 2011. J Arthroplast. 28: 116-119.

3. Cherian JJ, Jauregui JJ, Banerjee S, Pierce T, Mont MA (2015) What host factors affect aseptic loosening after THA and TKA? Clin Orthop Relat Res 473: 2700-2709.

4. Whyte MP (2010) Physiological role of alkaline phosphatase explored in hypophosphatasia. Ann NY Acad Sci 1192: 190-200.

5. Lee H, Dellatore SM, Miller WM, Messersmith PB (2007) Mussel-Inspired Surface Chemistry for Multifunctional Coatings. Science 318: 426-430.

6. Ryu JH, Messersmith PB, Lee H (2018) Polydopamine Surface Chemistry: A Decade of Discovery. ACS Appl Mater Interfaces 10: 7523-7540.

7. Schanze KS, Lee H, Messersmith PB (2018) Ten Years of Polydopamine: Current Status and Future Directions. ACS Appl Mater Interfaces 10: 7521-7522.

8. Alfieri ML, Micillo R, Panzella L, Crescenzi O, Oscurato SL, et al. (2018) Structural Basis of Polydopamine Film Formation: Probing 5,6-Dihydroxyindole-Based Eumelanin Type Units and the Porphyrin Issue. ACS Appl Mater Interfaces 10(9): 7670-7680.

9. Liebscher J, Mrówczyński R, Scheidt HA, Filip C, Hădade ND, et al. (2013) Structure of polydopamine: a never-ending story? Langmuir 29: 1053910548.

10. DeLory GE, King EJ (1945) A Sodium Carbonate-bicarbonate Buffer for Alkaline Phosphatases. Biochem 39: 16.

11. Andrea A, Mansell JP (2018) A Facile and Sensitive Colorimetric Approach to Confirming the Presence of Polydopamine Thin Films on (Bio) Material Surfaces. Regen Med Ther 2: 30-36.

12. Andrade MAR, Derradia R, Simãoa AMS, Millánb JL, Ramosa AP, (2019) Is alkaline phosphatase biomimeticaly immobilized on titanium able to propagate the biomineralization process? Arch Biochem Biophys 663: 192-198.

13. Low MG, Saltiel AR (1988) Structural and functional roles of glycosyl-phosphatidylinositol in membranes. Science 239: 268-275.

14. Burzio LA, Waite JH (2000) Cross-linking in adhesive quinoproteins: studies with model decapeptides. Biochemistry 39: 11147-11153.

15. Lee H. Rho J, Messersmith PB (2009) Facile conjugation of biomolecules onto surfaces via mussel adhesive protein inspired coatings. Adv Mater 21: $431-434$.

16. Chen KJ, Wang CH, Liao CW, Lee CK (2018) Recombinant fructosyl peptide oxidase preparation and its immobilization on polydopamine coating for colorimetric determination of HbA1c. Int J Biol Macromol 120: 325-331. 
17. Nijhuis AWG, Van Den Beucken JJJP, Jansen JA, Leeuwenburgh SCG (2013) In vitro response to alkaline phosphatase coatings immobilized onto titanium implants using electrospray deposition or polydopamine-assisted deposition. J Biomed Mater Res Part A 102A: 1102-1109.

ISSN: $2574-1241$

DOI: 10.26717/BJSTR.2020.27.004511

Jason P Mansell. Biomed J Sci \& Tech Res

(C) C) This work is licensed under Creative

Submission Link: https://biomedres.us/submit-manuscript.php
18. Kwon IS, Bettinger CJ (2018) Polydopamine nanostructures as biomaterials for medical applications. J Mater Chem B 6: 6895-6903.

$\begin{array}{ll}\text { BIOMEDICAL } & \text { Assets of Publishing with us } \\ \text { RESEARCHES } & \text { - Global archiving of articles } \\ & \text { - Immediate, unrestricted online access } \\ & \text { - Rigorous Peer Review Process } \\ & \end{array}$

